\title{
Regional distribution of fatiguing illnesses in the United States: a
} pilot study

\author{
Cynthia Bierl1, Rosane Nisenbaum¹, David C Hoaglin², Bonnie Randall², \\ Ann-Britt Jones ${ }^{1}$, Elizabeth R Unger ${ }^{1}$ and William C Reeves*1
}

\begin{abstract}
Address: ${ }^{1}$ Division of Viral and Rickettsial Diseases, National Center for Infectious Diseases, Centers for Disease Control and Prevention, Atlanta GA 30333, USA and 2Abt Associates Inc., Cambridge, MA 02138, USA

Email: Cynthia Bierl - cbie2606@gmp.usyd.edu.au; Rosane Nisenbaum - ran7@cdc.gov; David C Hoaglin - dave_Hoaglin@abtassoc.com; Bonnie Randall - bonnie_randall@abtassoc.com; Ann-Britt Jones - ABJones@rcsi.ie; Elizabeth R Unger - eru0@cdc.gov; William C Reeves* - wcr1@cdc.gov

* Corresponding author
\end{abstract}

Published: 04 February 2004

Population Health Metrics 2004, 2:1

This article is available from: http://www.pophealthmetrics.com/content/2/I/I

(C) 2004 Bierl et al; licensee BioMed Central Ltd. This is an Open Access article: verbatim copying and redistribution of this article are permitted in all media for any purpose, provided this notice is preserved along with the article's original URL.
Accepted: 04 February 2004
Received: 01 August 2003

\begin{abstract}
Background: Chronic fatigue syndrome (CFS) is a debilitating illness with no known cause or effective therapy. Population-based epidemiologic data on CFS prevalence are critical to put CFS in a realistic context for public health officials and others responsible for allocating resources.

Methods: We conducted a pilot random-digit-dialing survey to estimate the prevalence of fatiguing illnesses in different geographic regions and in urban and rural populations of the United States. This report focuses on 884 of 7,317 respondents 18 to 69 years old. Fatigued (440) and randomly selected non-fatigued (444) respondents completed telephone questionnaires concerning fatigue, other symptoms, and medical history.

Results: We estimated 12,186 per 100,000 persons 18 to 69 years of age suffered from fatigue lasting for at least 6 months (chronic fatigue), and I,197 per 100,000 described an illness that, though lacking clinical evaluation, met criteria for CFS (CFS-like). Chronic fatigue and CFS-like illness were more common in rural than in urban populations, although the differences were not significant. The prevalence of these fatiguing illnesses did not differ meaningfully among the four regions surveyed, and no significant geographic trends were observed.

Conclusions: This investigation estimated that nearly 2.2 million American adults suffer from CFSlike illness. The study also suggested the need to focus future investigations of fatigue on populations with lower incomes and less education. There was no evidence for regional differences in the occurrence of fatiguing illnesses.
\end{abstract}

\section{Background}

An understanding of the national prevalence and distribution of chronic fatigue syndrome (CFS) is fundamental to focusing etiologic research, targeting health-care and edu- cational programs, and estimating the secondary effects of debilitating fatigue on quality of life and on productivity. By ranking the burden of CFS and fatiguing illnesses among other national health concerns, a prevalence 
estimate can help frame appropriate health policy and raise political and public awareness for the concerns that emanate from this misunderstood and highly stigmatized syndrome.

Only two studies have examined population-based, random samples to estimate the prevalence of CFS and describe its demographic and socioeconomic characteristics. These studies surveyed the populations of Wichita, Kansas, and Chicago, Illinois, and determined similar estimates of CFS prevalence (142-560 per 100,000 persons) in the two cities $[1,2]$. In both studies, CFS primarily affected women, but the distribution of CFS by other descriptive characteristics differed between the two cities. These differences may reflect geographic and urbanization characteristics specific to the Wichita and Chicago metropolitan areas.

A national survey of CFS would be necessary to resolve questions raised by these two studies and to obtain information that could be generalized to CFS in the United States. Objectives of a national survey would include estimating the prevalence of CFS in urban and rural populations in different regions of the country; examining relationships between CFS and sex, age, race/ethnicity, and socioeconomic status in the different settings; and, exploring relationships among these variables and national patterns of health care use. Between July 2001 and January 2002, we conducted a pilot survey to determine feasibility and to test procedures for a national survey of CFS. The pilot study showed that clinical evaluation of persons with fatigue and accompanying symptoms characteristic of CFS (CFS-like illness) on a national level was logistically impractical and raised numerous issues of standardization and quality control. We elected not to pursue the full national survey. Nonetheless, the pilot survey collected important information concerning the epidemiology of chronic fatigue and CFS-like illness in a random sample of people selected from eight strata that comprised the contiguous United States.

\section{Methods}

This study adhered to human experimentation guidelines of the U.S. Department of Health and Human Services and complies with the Helsinki Declaration. All participants were volunteers who gave informed consent.

\section{Study design}

The survey used a stratified two-stage cluster design similar to that described in our previous study of fatiguing illnesses in the general population of Wichita, Kansas [1]. Briefly, the first stage of sampling selected a primary sampling unit (PSU) in each stratum, and the second stage drew a sample of telephone numbers for each PSU. Phase I of data collection screened households for individuals identified as fatigued for $\geq 1$ month. Phase II comprised detailed telephone interviews with individuals identified as fatigued and with a random sample of persons identified as non-fatigued. Phase III was a clinical evaluation of chronically fatigued subjects who, based on telephone interviews, had no exclusionary medical or psychiatric conditions and who met fatigue and symptom criteria of CFS (CFS-like).

\section{Sampling strategy}

To examine regional and metropolitan differences, we constructed strata from statistical areas defined by the US Census. Each of the four US Census regions (Northeast, Midwest, South, and West) was further stratified into metropolitan statistical areas (MSAs) and non-MSA counties. The U.S. Office of Management and Budget defines several categories of MSA, according to specific standards. In general terms an MSA has a core area containing a sizable population, together with adjacent communities that have a high degree of economic and social integration with that core. From each of the eight strata, we randomly selected a primary sampling unit (PSU), either an MSA or a non-MSA county (as appropriate). Because only one PSU was selected from each stratum, the results do not provide estimates of within-stratum variability. The eight PSUs selected were Buffalo-Niagara Falls, New York (Northeast urban), Chicago, Illinois (Midwest urban), Baton Rouge, Louisiana (South urban), Oakland, California (West urban), Franklin County, Pennsylvania (Northeast rural), Ripley County, Indiana (Midwest rural), Monroe County, Georgia (South rural), and Chaves County, New Mexico (West rural). For each PSU, 1,800 telephone numbers were randomly selected using the GENESYS Sampling System (Marketing Systems Group, Fort Washington, PA) and identifiable non-working and business numbers were removed. Advance letters, explaining the study, were sent to all households whose telephone number could be matched to a mailing address.

\section{Phase I - telephone screening interviews}

In each selected household, we screened an adult household informant who was at least 18 years old. The nature of the study was explained to the informant, who was requested to consent verbally before proceeding with the interview. The informant enumerated individual household members and reported on their age, sex, race, and fatigue status. Informants were asked whether any household members were currently suffering from severe fatigue, extreme tiredness, or exhaustion that had lasted 1 month or longer.

\section{Phase II - detailed telephone interviews}

Household residents older than 18 years identified with fatigue $\geq 1$ month were contacted for detailed interviews; parents were interviewed on behalf of adolescents (12 to 
17 years old). We also conducted detailed interviews on a random sample of non-fatigued adults 18 to 69 years old selected in a two-step process. First, households were randomly selected (with a probability of 0.25 ) to supply a non-fatigued person for a detailed interview. Second, if the household enumeration identified at least one age-eligible non-fatigued person in the household, a nonfatigued person was selected at random. Only one nonfatigued person could be selected from a household. A household in which a fatigued person was selected was eligible to have a non-fatigued person selected as well. However, in this study no household had both a fatigued and non-fatigued resident selected for interview.

As with the screening interview, the nature of the study was explained and subjects provided verbal informed consent before proceeding with the interview. The detailed interview included questions on fatigue (characteristics and duration), symptoms (occurrence, nature, and duration), demographics, and medical/psychiatric history. It also included the 12-item Short Form Health Survey ${ }^{\circledR}$ (QualityMetric, Inc., Lincoln, RI), which is designed to measure health-related quality of life.

\section{Case definitions}

CFS is an illness defined by symptoms and associated disability and by excluding medical diseases or psychiatric conditions that could explain them. There are no confirmatory physical signs or characteristic laboratory abnormalities. The current international CFS case definition [3] defines CFS as clinically evaluated, medically or psychiatrically unexplained, persistent or relapsing fatigue of at least 6 months duration that is not the result of ongoing exertion, is not substantially alleviated by rest, and results in substantial reduction in previous levels of occupational, educational, social, or personal activities. The fatigue must be accompanied by at least 4 of 8 symptoms that must have persisted or recurred during at least 6 consecutive months and cannot have predated the fatigue. Case defining symptoms include: 1) unusual post-exertional malaise of more than a day's duration following previously tolerated levels of mental or physical exertion; 2) unrefreshing sleep; 3 ) impaired short-term memory or concentration with substantial reduction in occupational, educational, social, or personal activities; 4) headaches of a new type, pattern, or severity; 5) muscle pain; 6) multijoint pain without swelling or redness; 7) sore throat; 8 ) tender cervical/axillary lymph nodes. The current CFS case definition relies entirely on self-reported symptoms and disability and specifies no standard measures. Recently an International Chronic Fatigue Syndrome Study Group has recommended resolutions for the major ambiguities [4].

This study relied on telephone interview to classify subjects. We used the CDC detailed telephone interview ques- tionnaire to identify and characterize fatigue, accompanying symptoms, and exclusionary conditions. This questionnaire (available from the authors on request) included minor modifications of one used over 4 years of surveillance in Wichita [1]. Individuals reporting fatigue lasting at least 6 months were classified as having chronic fatigue. We classified chronically fatigued respondents as having a CFS-likeillness if they had no medical or psychiatric exclusions identified during the interview and reported chronic fatigue that was not alleviated by rest and was accompanied at least 4 of the 8 CFS defining symptoms. Whether fatigue substantially interfered with work, educational, or personal activities was not assessed in the detailed telephone interviews. Classification as CFSrequires a complete physical examination to accurately detect medical or psychiatric conditions that subjects may not recount on interview. Fatigue categories were analyzed as discrete groups (chronic fatigueand CFSlike), and each subject was counted only in the most restrictive category that applied.

\section{Statistical analyses}

Prevalence estimates were based on weighted data. Each household received a base sampling weight that reflected the probability of selection of the PSU and selection of the household telephone number within the PSU. The final household sampling weight incorporated adjustments for multiple residential lines, non-response, and households without telephones. For non-fatigued individuals who completed detailed interviews, the person-level weight was determined by the household weight and the person's probability of selection. For fatigued individuals, all of whom were selected with certainty, the person-level weight for the detailed interview equaled the household weight. Weighted prevalences and weighted Pearson chisquared and Wald statistics were calculated by using STATA 7.0 (Stata Corporation, College Station, TX). Confidence intervals for prevalences were constructed by using a logit transformation (hence the lower endpoint was always greater than 0 ).

\section{Results \\ Interview sample}

Of the 14,405 telephone numbers in the sample, 7,573 (52.6\%) were ineligible because they belonged to businesses, were not working, or were cellular phones. Residential status could not be determined for 4,018 (27.9\%) of the numbers: 791 (5.5\%) because all attempts produced no contact (i.e., the number was busy, was temporarily disconnected, or rang but was never answered), 278 $(1.9 \%)$ because the attempts reached only an answering machine, and 2,949 (20.5\%) because of some other outcome (mainly the person answering refused to participate before household status could be determined). The remaining 2,809 (19.5\%) numbers were residential, and 
Table I: Weighted proportion of descriptive characteristics in mutually exclusive categories of fatiguing illness.

\begin{tabular}{|c|c|c|c|c|}
\hline Characteristic & $\%$ Non-fatigued $n=444$ & $\%$ Chronic fatigue $n=338$ & $\%$ CFS-like $n=49$ & $\%$ Total \\
\hline Female & 57 & 66 & 70 & 58 \\
\hline White & 81 & 89 & 88 & 82 \\
\hline Non-Hispanic & 95 & 92 & 99 & 95 \\
\hline \multicolumn{5}{|l|}{ Age Group (years) } \\
\hline $18-29$ & 22 & 11 & 18 & 21 \\
\hline $30-39$ & 21 & 15 & 19 & 20 \\
\hline $40-49$ & 30 & 33 & 27 & 30 \\
\hline $50-59$ & 17 & 24 & 24 & 18 \\
\hline $60-69$ & 10 & 18 & 13 & 11 \\
\hline \multicolumn{5}{|l|}{ Household income } \\
\hline$\leq \$ 40,000^{*}$ & 34 & 50 & 49 & 37 \\
\hline \multicolumn{5}{|l|}{ Educational level* } \\
\hline$\leq$ High school graduate & 39 & 61 & 58 & 43 \\
\hline
\end{tabular}

* Distribution differs significantly among categories. Tested by design-based Pearson chi-squared test $P<.00 \mathrm{I}$ ).

2,728 (97.1\%) of these households completed screening interviews. These 2,728 households contained a total of 7,317 individuals; 744 had fatigue lasting $\geq 1$ month, and 6,573 were non-fatigued.

Eighty-four of the 744 individuals identified as fatigued were ineligible for the detailed interview (outside age range, language barrier) and 161 (21.6\%) refused to participate. The remaining 455 persons identified as fatigued in the screening interview completed detailed interviews; 440 were between the ages of 18 and 69 and are included in this report. Six hundred of the 6,573 non-fatigued individuals were selected for a detailed interview; 444 nonfatigued individuals between the ages of 18 and 69 completed detailed interviews and are included in this report. Eligibility and refusal rates were similar for non-fatigued and fatigued respondents.

The 884 respondents completing detailed interviews represent a weighted population of $181,000,000$ persons in the eight strata; the 444 non-fatigued respondents represent 153,000,000 non-fatigued persons in the survey population. Of the 440 fatigued respondents, 53 reported fatigue of less than 6 months duration, 338 were classified as having chronic fatigue, and 49 satisfied the criteria for CFS-like illness, representing an estimated 22 million and 2.2 million persons, respectively.

\section{Population characteristics}

Table 1 shows the weighted population characteristics by fatigue category estimated from the interview sample. Demographics were similar to those reported by the US 2000 Census, except that the current study surveyed a higher proportion of white non-Hispanic individuals. The survey population was predominantly white $(82 \%)$ and non-Hispanic (95\%). The mean age of respondents was
42 years, 37\% reported an income of $\leq \$ 40,000$ per year, and $57 \%$ reported education beyond high school.

As seen in Table 1, the distributions of sex, race, and ethnicity did not differ significantly among categories of fatiguing illness. The proportion of individuals reporting a household income of $\leq \$ 40,000$ or education beyond high school was significantly different among fatigue categories $(\mathrm{P}<.0001$ and $=.0001$, respectively $)$. In general, lower income and less education were associated with higher prevalence of fatiguing illness.

Table 2 lists the weighted proportions of medical and psychiatric conditions identified in the detailed interviews. A higher proportion of individuals in the fatigued sample (43.3\%) reported medical and psychiatric exclusions than in the non-fatigued sample $(11.2 \%)(P<.0001)$.

\section{Prevalence estimates}

Table 3 presents overall prevalence estimates of fatiguing illnesses and estimates by urban, rural, and regional location. Chronic fatigue affected 12,186 per 100,000 persons; we estimate that 1,197 people per 100,000 persons suffered from CFS-like illness. Both chronic fatigue and CFS-like illness were more common in rural than in urban populations, although the differences were not significant. There were no significant differences in prevalence estimates by geographic regions.

Table 4 gives prevalence estimates of fatiguing illnesses by demographic characteristics. Although women had much higher prevalence of chronic fatigue and CFS-like illness than men, the differences between these estimates were not significant. The prevalence of chronic fatigue in whites was significantly higher than in non-white individuals $(P$ $=.024)$. The prevalence of reported chronic fatigue was 
Table 2: Weighted proportion of medical and psychiatric exclusionary conditions in non-fatigued and fatigued samples.

\begin{tabular}{lcc}
\hline Medical or Psychiatric Exclusionary Condition & \% Non-fatigued & \%atigued* \\
\hline Any exclusionary condition & 11.16 & 43.26 \\
Rheumatoid arthritis & 1.86 & 13.13 \\
Lupus/Sjögren's syndrome & 0.00 & 2.70 \\
Pregnancy & 6.48 & 7.66 \\
Heart failure/fluid in lungs & 1.95 & 6.35 \\
Hepatitis/cirrhosis & 0.41 & 4.20 \\
Immunodeficiency disease & 0.00 & 1.63 \\
Multiple sclerosis & 0.08 & 2.54 \\
Heart condition limiting walking & 0.16 & 5.52 \\
Heart attack & 1.70 & 3.66 \\
Organ transplant & 0.91 & 0.36 \\
Stroke & 0.16 & 2.95 \\
Cancer, including lymphoma or leukemia & 2.92 & 6.22 \\
Alcohol/drug dependency & 1.17 & 4.73 \\
Manic depressive disorder & 1.05 & 0.11 \\
Anorexia/bulimia & 0.52 & 1.97 \\
Schizophrenia & & 2.58 \\
\hline
\end{tabular}

* Fatigued includes subjects fatigued for at least one month

Table 3: Weighted prevalence (per 100,000 persons) of fatiguing illnesses nationally and by urban, rural, and regional location.

\begin{tabular}{|c|c|c|c|c|}
\hline \multirow{3}{*}{$\begin{array}{l}\text { Location } \\
\text { Overall }\end{array}$} & \multicolumn{4}{|c|}{ Prevalence (95\% Confidence Interval) } \\
\hline & \multicolumn{2}{|c|}{ Chronic Fatigue } & \multicolumn{2}{|c|}{ CFS-like Illness } \\
\hline & 12186 & $(9930-14870)$ & 1197 & $(810-1770)$ \\
\hline Urban & 11480 & $(8780-14880)$ & 1040 & $(600-1770)$ \\
\hline Rural & 14260 & $(I I I 40-18080)$ & 1670 & $(1030-2690)$ \\
\hline \multicolumn{5}{|l|}{ Region } \\
\hline Northeast & 12490 & $(8340-18300)$ & 1900 & $(960-3750)$ \\
\hline Midwest & 14360 & $(9490-21160)$ & 990 & $(430-2270)$ \\
\hline South & 11040 & $(7850-15310)$ & 970 & $(470-1980)$ \\
\hline West & 11690 & $(6930-19040)$ & 1160 & $(460-2900)$ \\
\hline
\end{tabular}

significantly higher in the 40 to 49 year $(P=.03), 50$ to 59 year $(P=.006)$, and 60 to 69 year $(P=.012)$ age groups than in the 18 to 29 year age group.

Individuals with incomes of $\leq \$ 40,000$ per year were more likely to report chronic fatigue and CFS-like illness than respondents with higher incomes. The prevalence of chronic fatigue $(P=.015)$ was significantly higher in the low-income category. The prevalences of chronic fatigue and CFS-like illness were highest in individuals who reported no more than a high school education; but the only significant difference was in the prevalence of chronic fatigue $(P=.002)$.

\section{Discussion}

This population-based pilot survey of US households estimates that 12,186 per 100,000 adults in the US suffer severe chronic fatigue of $>6$ months, and 1,197 have a CFS-like illness. The prevalence of CFS-like illness was similar to that in the Wichita population $(1,607$ per $100,000)[1]$, which used a similar sampling strategy and virtually identical telephone interviews. Age distributions of CFS-like illness were similar in both this and the Wichita study, the highest risk occurring in the 40 to 59 year age range. Finally, women were 2.3 times more likely than men to be identified with CFS-like illness in both Wichita and this pilot survey. However, in this survey the difference was not statistically significant, most likely reflecting small sample size. 
Table 4: Weighted prevalence (per 100,000 persons) of fatiguing illnesses by demographic characteristics.

\begin{tabular}{|c|c|c|c|c|}
\hline \multirow[t]{2}{*}{ Characteristic } & \multicolumn{4}{|c|}{ Prevalence (95\% Confidence Interval) } \\
\hline & \multicolumn{2}{|c|}{ Chronic Fatigue } & \multicolumn{2}{|c|}{ CFS-like IIIness } \\
\hline \multicolumn{5}{|l|}{ Sex } \\
\hline Male & 9900 & $(7050-13750)$ & 860 & $(420-1760)$ \\
\hline Female & 13810 & $(10620-17770)$ & 1440 & $(900-2290)$ \\
\hline \multicolumn{5}{|l|}{ Race } \\
\hline Non-white & $7260^{*}$ & $(4570-12440)$ & 790 & $(270-2290)$ \\
\hline White & $13 \mid 40$ & $(10520-16290)$ & 1280 & $(840-1950)$ \\
\hline \multicolumn{5}{|l|}{ Age (yrs) } \\
\hline $18-29$ & 6440 & $(3540-11420)$ & 1030 & $(350-2950)$ \\
\hline $30-39$ & 8870 & $(5660-13620)$ & 1120 & $(520-2390)$ \\
\hline $40-49$ & $13310^{*}$ & $(9060-19140)$ & 1050 & $(540-2050)$ \\
\hline $50-59$ & $16300^{*}$ & $(I / 240-23040)$ & 1630 & $(700-3750)$ \\
\hline $60-69$ & $19590 *$ & $(I / 790-30760)$ & 1370 & $(420-4320)$ \\
\hline \multicolumn{5}{|c|}{ Household income } \\
\hline$\leq \$ 40,000$ & $17180 *$ & $(12960-22410)$ & 1470 & (790-2750) \\
\hline$>\$ 40,000$ & 9990 & $(7 / 20-13850)$ & 920 & $(510-1650)$ \\
\hline \multicolumn{5}{|l|}{ Educational level } \\
\hline$\leq$ High school & $17030 *$ & $(12550-22710)$ & 1650 & (940-2880) \\
\hline$\geq$ College & 8280 & $(6310-10790)$ & 880 & $(500-1550)$ \\
\hline
\end{tabular}

* Difference between categories of characteristic is significant by survey-adjusted Wald test $(P<.05)$

Previous investigations linking the distribution of fatiguing illness according to race/ethnicity were not supported in this study [1,2]. Similarly, we found no evidence for differences in the prevalence of fatiguing illness by urban versus rural or by geographic region. However, in contrast to previous studies, we found lower income and less education to be the strongest predictive factors for fatiguing illness.

These results are preliminary findings and must be interpreted conservatively. The primary objective of the pilot study was to determine feasibility and test procedures for a national survey of CFS. Thus we selected only one PSU from each stratum, whereas a full-scale national survey might involve a total of 100 or more PSUs. The selected sample may not be large enough to detect various possible demographic differences in CFS-like estimates. Also, persons with CFS-like illness must undergo a clinical evaluation to identify exclusionary medical and psychiatric conditions and confirm classification as CFS. Clinical evaluation proved difficult in terms of logistics and quality control. Only 7 of the 49 subjects with CFS-like illness completed a clinical evaluation, and none had CFS.

Interpretation of the findings must also consider that there are no diagnostic signs or laboratory abnormalities so CFS is diagnostic solely on symptoms [3]. Symptom criteria of the 1994 CFS research case definition are based on self-report and there are no published standardized and validated instruments that identify CFS in a manner similar to the CIDI, DIS, or SCID for DSM-IV conditions [4]. We obtained information to classify subjects as CFSlike with an instrument designed by the CDC CFS Research Group and used in previous CFS surveillance studies. As noted, prevalence of CFS-like illness in the national pilot study was similar to that in earlier CDC studies that utilized the same instrument. We have also used data from our most recent longitudinal surveillance study [1] to perform exemplary analyses to validate the instrument by comparing responses to the telephone survey questions with scores in the Multidimensional Fatigue Inventory [5]. These analyses confirmed adequate validity of the instrument [CDC unpublished].

Interpretation of the findings must also consider that CFS is an exclusionary diagnosis based on symptoms for which a medical or psychiatric explanation cannot be found [3]. Subjects with exclusionary conditions reported during the telephone interview were not classified as CFSlike. However, to confirm diagnosis of CFS, persons who meet symptom criteria on interview must be clinically evaluated to identify medical or psychiatric conditions not detected by interview. Just over 40 percent of subjects with CFS-like illness detected in community surveys have an exclusionary medical or psychiatric condition following clinical evaluation $[1,2]$. 
Finally, interpretation of the findings must consider epidemiologic limitations in defining prevalence of an illness such as CFS. This study attempted to estimate the point prevalence of CFS-like illness (a surrogate for CFS). That is the probability that an individual fulfilled case definition criteria at the time of the survey. CFS presents a complex and intermittent pattern of illness over time and persistent CFS is the exception not the rule [6]. In population surveys approximately 40 percent of persons with CFS-like illness who are referred for clinical evaluation do not meet all fatigue and symptom severity criteria of the case definition when evaluated clinically and thus cannot be considered prevalent cases $[1,2]$. Most of them likely represent temporary remissions of the illness [6] but there are no straightforward ways to consider this in prevalence estimates.

\section{Conclusions}

In conclusion, this investigation suggests that 2.2 million American adults between the ages of 18 and 69 years suffer from CFS-like illness. We did not find evidence for regional differences in the prevalence of fatiguing illnesses but instead found that fatiguing illnesses are of overall national importance. Most persons with CFS-like illness found in this study had lower incomes and less education than the non-fatigued population, suggesting social risk factors (such as stress) may be important in the etiology of CFS. This has important implications for public health officials, health care providers, and the public in general. The methodology and findings from this study should be of interest to those studying CFS and with responsibilities for health care in other countries.

\section{Competing interests}

None declared.

\section{Authors Contributions}

CB had primary responsibility for data analysis and interpretation of results, and drafted the manuscript. RN participated in the conception, design and implementation of the study, helped to oversee its conduct, supervised and collaborated in data analysis, collaborated in interpreting the results, and collaborated in drafting the manuscript. DCH collaborated in the study design, was responsible for sampling strategy, collaborated in data analysis/interpretation, and helped to draft the manuscript. BR contributed to the study design, was responsible for implementation and conduct of the study, and participated in interpretation of the results. ABJ had major responsibility for study design and implementation, overseeing conduct of the fieldwork, interpretation of results, and collaborated in drafting the manuscript. ERU participated in study design, interpretation of results, and drafting the manuscript. WCR conceived of the study, participated in its design, implementation, and conduct, collaborated in interpreta- tion of the results, and helped to draft the manuscript. All authors read and approved the final manuscript.

\section{References}

I. Reyes M, Nisenbaum R, Hoaglin DC, Emmons C, Stewart G, Randall B, Unger ER, Stewart J, Abbey S, Jones J, Gantz N, Minden S, Reeves WC: Prevalence and incidence of chronic fatigue syndrome in Wichita, Kansas. Arch Intern Med 2003, I63:I530-I536.

2. Jason LA, Richman JA, Rademaker AW, Jordan KM, Plioplys AV, Taylor RR, McCready W, Huang CF, Plioplys S: A community-based study of chronic fatigue syndrome. Arch Intern Med I999, I 59:2 I 29-2 I 37.

3. Fukuda K, Straus SE, Hickie I, Sharpe MC, Dobbins JG, Komaroff A: The chronic fatigue syndrome: a comprehensive approach to its definition and study. Ann Intern Med 1994, I 2 I:953-959.

4. Reeves WC, Lloyd A, Vernon SD, Klimas N, Jason LA, Bleijenberg G, Evengard B, White PD, Nisenbaum R, Unger ER: Identification of ambiguities in the 1994 chronic fatigue syndrome research case definition and recommendations for resolution. $B M C$ Health Serv Res 2003, 3:25.

5. Smets EMA, Garssen B, Bonke B, de Haes JCJM: The multidimensional fatigue inventory (MFI) psychometric qualities of an instrument to assess fatigue. J Psychosom Res 1995, 39:315-325.

6. Nisenbaum R, Jones JF, Unger ER, Reyes M, Reeves WC: A population-based study of the clinical course of chronic fatigue syndrome. Health Quality of Life Outcomes 2003, I:49.

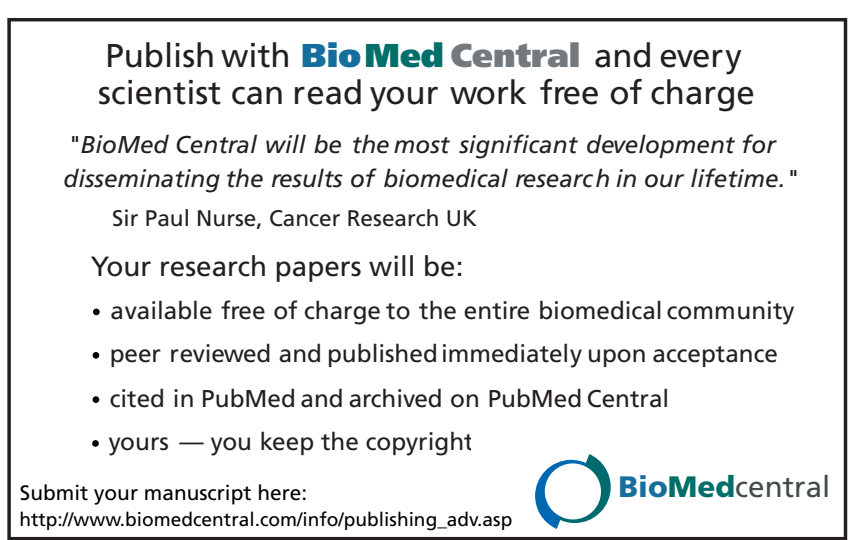

